\title{
AN ELEMENTARY PROOF OF SOME CHARACTER SUM IDENTITIES OF APOSTOL
}

\author{
by BRUCE C. BERNDT
}

(Received 19 May, 1971)

Let $\chi$ denote a primitive character modulo $k$. Using two different representations for Dirichlet $L$-functions, Apostol [1] recently derived a representation for

$$
M_{m}(\chi)=\sum_{r=1}^{k-1} \chi(r) r^{m}
$$

involving the sums

$$
T_{m}(\bar{\chi})=\sum_{r=1}^{k-1} \bar{\chi}(r) \cot ^{m}(\pi r / k),
$$

where $m$ is a positive integer. Furthermore, if $\chi(r)=(r \mid p)$, the residue class character modulo the odd prime $p$, he derived a representation for $M_{m}(\chi)$ involving the sums

$$
S_{m}=\sum_{r=1}^{p-1} \cot ^{m}\left(\pi r^{2} i p\right) .
$$

A completely elementary proof of these identities is given here.

We shall use the simple facts,

$$
\sum_{r=1}^{k} e^{2 \pi i r h / k}=\left\{\begin{array}{lll}
k, & \text { if } & k \mid h, \\
0, & \text { if } & k \nmid h,
\end{array}\right\}
$$

and

$$
\sum_{r=1}^{k} \chi(r)=0
$$

Let $G(m, \chi)$ denote the Gaussian sum

$$
G(m, \chi)=\sum_{r=1}^{k-1} \chi(r) e^{2 \pi i r m / k},
$$

and put $G(\chi)=G(1, \chi)$. We shall need the factorization theorem for Gaussian sums associated with a primitive character [2, p. 67],

$$
G(m, \bar{\chi})=\chi(m) G(\bar{\chi}) .
$$

Throughout the sequel, $\chi$ denotes a primitive character.

THEOREM 1. If $n$ is a positive integer, define

$$
f(\chi, n)=\sum_{h=1}^{k-1} \bar{\chi}(h)\left\{\frac{e^{2 \pi i h / k}}{1-e^{2 \pi i h / k}}\right\}^{n} .
$$


Then

$$
(-k)^{n} f(\chi, n)=G(\bar{\chi}) \sum_{j_{1}, j_{2}, \ldots, j_{n}=1}^{k} j_{1} j_{2} \ldots j_{n} \chi\left(j_{1}+j_{2}+\ldots+j_{n}\right)
$$

Proof. If $k \nmid h$, for any positive integer $r$,

$$
\sum_{j=1}^{r} e^{2 \pi i j h / k}=\frac{e^{2 \pi i h / k}-e^{2 \pi i(r+1) h / k}}{1-e^{2 \pi i h / k}} .
$$

Hence

$$
f(\chi, n)=\sum_{h=1}^{k-1} \bar{\chi}(h) \prod_{m=1}^{n}\left\{\sum_{j_{m}=1}^{r_{m}} e^{2 \pi i j_{m} h / k}+\frac{e^{2 \pi i\left(r_{m}+1\right) h / k}}{1-e^{2 \pi i h / k}}\right\},
$$

where $1 \leqq r_{m} \leqq k, 1 \leqq m \leqq n$. Now sum both sides of (4) over $r_{m}, 1 \leqq r_{m} \leqq k, 1 \leqq m \leqq n$. Upon using (1), we find that

$$
k^{n} f(\chi, n)=\sum_{n=1}^{k-1} \bar{\chi}(h) \sum_{r_{1}=1}^{k} \ldots \sum_{r_{n}=1}^{k} \sum_{j_{1}=1}^{r_{1}} \ldots \sum_{j_{n}=1}^{r_{n}} e^{2 \pi i\left(j_{1}+j_{2}+\ldots+j_{n}\right) h / k} .
$$

Invert the order of summation on $r_{m}$ and $j_{m}(1 \leqq m \leqq n)$ and use (1). We obtain

$$
\begin{aligned}
k^{n} f(\chi, n) & =\sum_{h=1}^{k-1} \bar{\chi}(h) \sum_{j_{1}=1}^{k}\left(k-j_{1}+1\right) e^{2 \pi i j_{1} h / k} \ldots \sum_{j_{n}=1}^{k}\left(k-j_{n}+1\right) e^{2 \pi i j_{n} h / k} \\
& =(-1)^{n} \sum_{j_{1}, j_{2}, \ldots, j_{n}=1}^{k} j_{1} j_{2} \ldots j_{n} \sum_{h=1}^{k-1} \bar{\chi}(h) e^{2 \pi i\left(j_{1}+j_{2}+\ldots+j_{n}\right) h / k} \\
& =(-1)^{n} \sum_{j_{1}, j_{2}, \ldots, j_{n}=1}^{k} j_{1} j_{2} \ldots j_{n} G\left(j_{1}+j_{2}+\ldots+j_{n}, \bar{\chi}\right) \\
& =(-1)^{n} G(\bar{\chi}) \sum_{j_{1}, j_{2}, \ldots, j_{n}=1}^{k} j_{1} j_{2} \ldots j_{n} \chi\left(j_{1}+j_{2}+\ldots+j_{n}\right),
\end{aligned}
$$

by (3), and the proof is complete.

For $1 \leqq m \leqq 4$, Apostol [1] expressed $M_{m}(\chi)$ as a linear combination of $T_{1}(\bar{\chi}), \ldots, T_{m}(\bar{\chi})$. From his calculations it became clear that the same result is valid for an arbitrary positive integer $m$. These representations for $M_{m}(\chi)$ can be derived from Theorem 1 . We shall work out the details for the first two examples.

Example 1. If $x$ is not an integer, then

$$
\frac{1}{2} i \cot \pi x=\frac{1}{2}+e^{2 \pi i x} /\left(1-e^{2 \pi i x}\right) .
$$

Hence, by the use of Theorem 1 and (2), we have

i.e.,

$$
\frac{1}{2} i k T_{1}(\bar{\chi})=k f(\chi, 1)=-G(\bar{\chi}) \sum_{j=1}^{k} \chi(j) j,
$$

$$
G(\bar{\chi}) M_{1}(\chi)=-\frac{1}{2} i k T_{1}(\bar{\chi})
$$


Example 2. Upon using (5) and (2), we find that

$$
-\frac{1}{4} k^{2} T_{2}(\bar{\chi})=k^{2} f(\chi, 1)+k^{2} f(\chi, 2) .
$$

To evaluate $f(\chi, 2)$ we use Theorem 1. Letting $j_{2}=r-j_{1}$ and $j_{1}=j$, we obtain, with the use of (2),

$$
k^{2} f(\chi, 2)=G(\bar{\chi}) \sum_{j=1}^{k} j \sum_{r=j+1}^{j+k} r \chi(r) .
$$

If we invert the order of summation, we find that

$$
\begin{aligned}
k^{2} f(\chi, 2) & =G(\bar{\chi})\left\{\sum_{r=2}^{k} r \chi(r) \sum_{j=1}^{r-1} j+\sum_{r=k+1}^{2 k} r \chi(r) \sum_{j=r-k}^{k} j\right\} \\
& =G(\bar{\chi})\left\{\sum_{r=2}^{k} \frac{1}{2} r^{2}(r-1) \chi(r)+\sum_{r=1}^{k}(r+k) \chi(r)\left[\frac{1}{2} k(k+1)-\frac{1}{2} r(r-1)\right]\right\} \\
& =G(\bar{\chi})\left\{\frac{1}{2} k^{2} M_{1}(\chi)+k M_{1}(\chi)-\frac{1}{2} k M_{2}(\chi)\right\},
\end{aligned}
$$

upon simplification and the use of (2). We now substitute (7) into (6) and use the results of Example 1. After a little simplification we arrive at

$$
G(\bar{\chi}) M_{2}(\chi)=-\frac{1}{2} i k^{2} T_{1}(\bar{\chi})+\frac{1}{2} k T_{2}(\bar{\chi}) .
$$

Next, we show that the second class of identities given by Apostol [1] can be derived in an elementary manner.

THEOREM 2. If $p$ is an odd prime and $n$ is a positive integer, define

$$
g(p, n)=\sum_{r=1}^{p-1}\left\{\frac{e^{2 \pi i r^{2} / p}}{1-e^{2 \pi i r^{2} / p}}\right\}^{n}
$$

If $\chi(r)=(r \mid p)$, then

$$
(-p)^{n} g(p, n)=G(\chi) \sum_{j_{1}, j_{2}, \ldots . j_{n}=1}^{p} j_{1} j_{2} \ldots j_{n} \chi\left(j_{1}+j_{2}+\ldots+j_{n}\right)+\sum_{h=1}^{p-1}\left\{\sum_{j=1}^{p} j e^{2 \pi i j h / p}\right\}^{n} .
$$

Since

$$
\sum_{j=1}^{p} j e^{2 \pi i j h / p}=\frac{1}{2} p\{1-i \cot (\pi h / p)\}
$$

the second expression on the right side of (8) may be written as

$$
\left(\frac{1}{2} p\right)^{n} \sum_{h=1}^{p-1}\{1-i \cot (\pi h / p)\}^{n}
$$

Proof. Proceeding as in the proof of Theorem 1, we arrive at

$$
(-p)^{n} g(p, n)=\sum_{j_{1}, j_{2}, \ldots, j_{n}=1}^{p} j_{1} j_{2} \ldots j_{n} \sum_{r=1}^{p-1} e^{2 \pi i\left(j_{1}+j_{2}+\ldots+j_{n}\right) r^{2} / p}
$$


Since each congruence $r^{2} \equiv h(\bmod p)$ has either 0 or 2 solutions modulo $p$, we have

upon the use of (3).

$$
\begin{aligned}
(-p)^{n} g(p, n) & =2 \sum_{j_{1}, j_{2}, \ldots, j_{n}=1}^{p} j_{1} j_{2} \ldots j_{n} \sum_{\substack{h=1 \\
(h \mid p)=1}}^{p-1} e^{2 \pi i\left(j_{1}+j_{2}+\ldots+j_{n}\right) h / p} \\
& =\sum_{j_{1}, j_{2}, \ldots, j_{n}=1}^{p} j_{1} j_{2} \ldots j_{n} \sum_{h=1}^{p-1}\{(h \mid p)+1\} e^{2 \pi i\left(j_{1}+j_{2}+\ldots+j_{n}\right) h / p} \\
& =G(\chi) \sum_{j_{1}, j_{2}, \ldots, j_{n}=1}^{p} j_{1} j_{2} \ldots j_{n} \chi\left(j_{1}+j_{2}+\ldots+j_{n}\right)+\sum_{h=1}^{p-1}\left\{\sum_{j=1}^{p} j e^{2 \pi i j h / p}\right\}^{n},
\end{aligned}
$$

Theorem 2 may be employed to show that $M_{m}(\chi)$ can be written as the sum of a polynomial in $p$ and a linear combination of $S_{1}, \ldots, S_{m}$. We shall work out the details for the first two cases.

Example 3. Upon the use of (5) and Theorem 2,

or, upon simplification,

$$
\begin{aligned}
\frac{1}{2} i p S_{1} & =\frac{1}{2} p(p-1)+p \dot{g}(p, 1) \\
& =\frac{1}{2} p(p-1)-G(\chi) M_{1}(\chi)-\sum_{j=1}^{p} j \sum_{h=1}^{p-1} e^{2 \pi i j h / p} \\
& =\frac{1}{2} p(p-1)-G(\chi) M_{1}(\chi)+\sum_{j=1}^{p-1} j-p(p-1),
\end{aligned}
$$

$$
G(\chi) M_{1}(\chi)=-\frac{1}{2} i p S_{1} .
$$

Example 4. Employing (5) and the value of $g(p, 1)$ from Example 3, we have

$$
-\frac{1}{4} S_{2}=\frac{1}{2} i S_{1}-\frac{1}{4}(p-1)+g(p, 2) \text {. }
$$

Using Theorem 2 and Example 2, we find after simplification that

$$
G(\chi) M_{2}(\chi)=\frac{1}{2} p S_{2}-i p^{2} S_{1}-\frac{1}{2} p(p-1)+\frac{2}{p_{j_{1}}} \sum_{j_{2}=1}^{p} j_{1} j_{2} \sum_{h=1}^{p-1} e^{2 \pi i\left(j_{1}+j_{2}\right) h / p} .
$$

This last expression may be evaluated by separating out the terms when $j_{1}+j_{2}=p$ or $2 p$. Upon doing this, we find that the triple sum in (9) becomes

$$
\text { (p-1) } \sum_{\substack{j_{1}, j_{2}=1 \\ j_{1}+j_{2}=p}}^{p} j_{1} j_{2}+p^{2}(p-1)-\sum_{\substack{j_{1}, j_{2}=1 \\ j_{1}+j_{2} \neq p, 2 p}}^{p} j_{1} j_{2}=-p^{4} / 12+p^{3} / 3-5 p^{2} / 12 .
$$

Upon substituting the above into (9) and simplifying, we obtain

$$
G(\chi) M_{2}(\chi)=\frac{1}{2} p S_{2}-\frac{1}{2} i p^{2} S_{1}-\frac{1}{6} p(p-1)(p-2) .
$$

\section{REFERENCES}

1. T. M. Apostol, Dirichlet $L$-functions and character power sums, J. Number Theory 2 (1970), 223-234.

2. H. Davenport, Multiplicative number theory (Chicago, 1967).

UNIVERSITY OF ILLINOIS

URBANA, ILLINOIS 